\title{
Reduction of Energy Sweep of the ETA-II Beam
}

\author{
W.E. Nexsen, S.L. Allen, F.W. Chambers, R.A. Jong, A.C. Paul, \\ S.E. Sampayan, and W.C. Tumer
}

This paper was prepared for submittal to the 1991 IEEE Particle Accelerator Conference May 6-9, 1991, San Francisco, CA

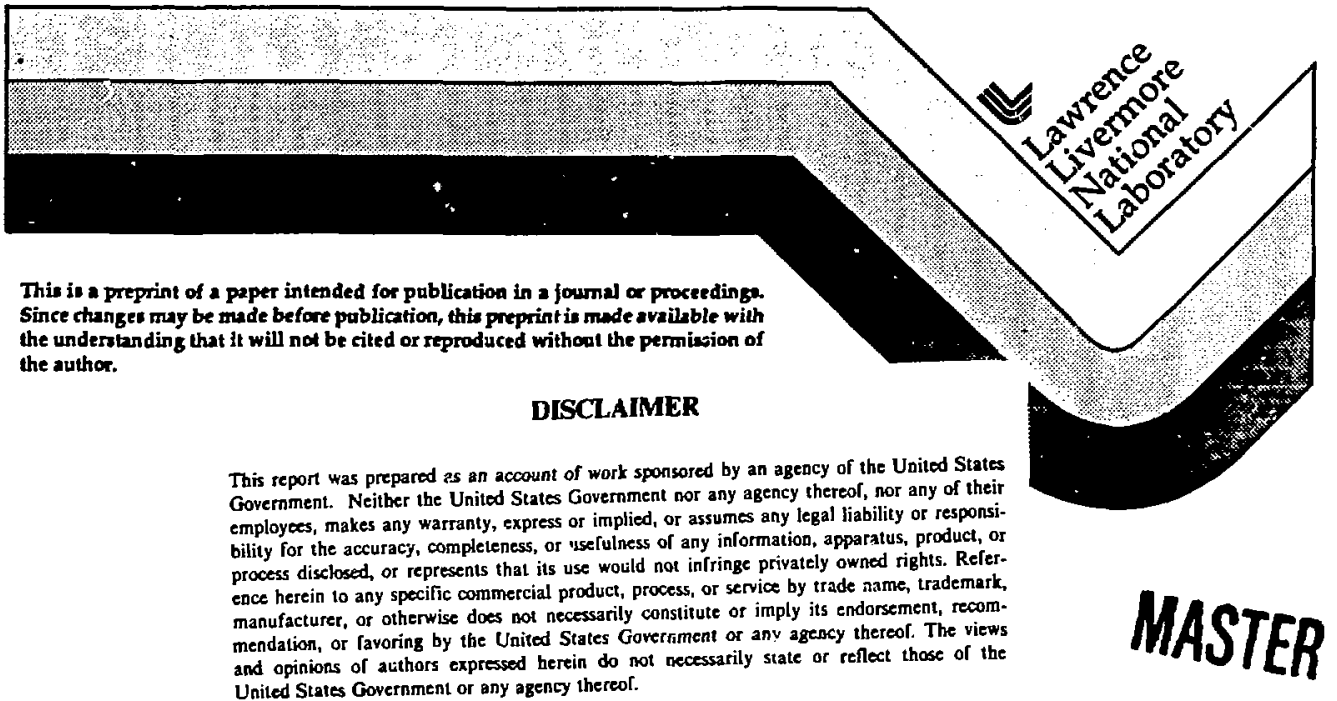




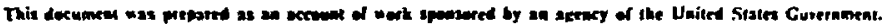

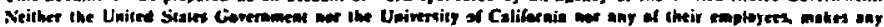

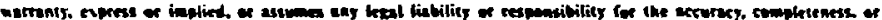

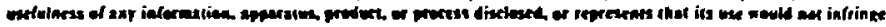

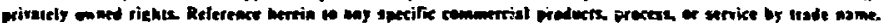

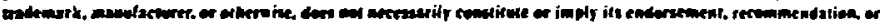

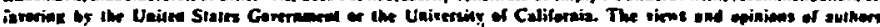

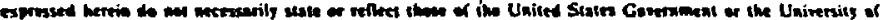

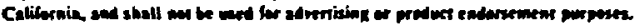

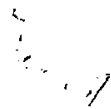




\title{
Reduction of Energy Sweep of the ETA-II Beam*
}

\author{
W. Nexsen, SL. Allen, F.W. Chanbers, R.A. Jong, A.C. PaW, S.E. Sampayan and W.C. Tumer \\ Lawerence Livermare National Laboratory \\ University of California \\ Livermore, CA 94550
}

\section{Abstract -}

The ETA-II electron beam will be used 10 drive a high power microwave frequency FEL for plasma hepting experiments. For maximum FEL output power the beam energy st the entrance to the wiggler should be within $\pm 1 \%$ of the wiggler resonance value. In ivitial operations the ETA-II beam energy sayed within this range for a maximum time of less than $13 \mathrm{~ns}$. Much of the energy variation was due to the design of the pulsed power feeds to the accelerator induction cells. A new multicable pulsed power foed design was rested in a shortened version of ETA-II where it exlended the time during which the beam energy stayed within the $\pm 1 \%$ limits to greater than 40 ns. These design changes are now being incorporated into the full accelerator.

\section{INTRODUCTHON}

The Experimental Test Accelerator I (ETA-II) facility is funded to develop and demonstrate the electron induction linac lechnology necessary for driving FELs al high average power [1]. The facility consists of the ETA-II sccelentor, (the first induction linac desigried specifically to drive an FEL), and several test stands for studying cathode brightness and poisoning, ferrite response, pulse-power feeds, and the operation of magnetic switches at high repetition frequency (pri). The design changes described in this report, which were terted on a shortened version of the accelentor, are now being incorparited in the full machine. When completed ETA-II will be able to supply 70 ns full-widu-helf-meximum pulses of 2 $3 \mathrm{kA}$ beam current, 6-7 Mev energy, in Iffy pulse bursts at 5 kHz prf with a burst repitition mute of $0.5 \mathrm{~Hz}$. As a technology demonstration we intend to use ETA-II wo dive $=140 \mathrm{GHz}$ FEL, the Intense Microwave Protetype (IMP) amplifier system [2]. whose output will be used for plasma heating experiments on the LLNL Microwave Tokanal Experiment (MIX).

The IMP system consists of a inicrowave oscillator, a quasi-optic coupler which injects the oscillator output coaxially with the beam and a wiggler which couples bean energy wo the electomagnetic field. The bigh magnetic field and wide unnbility capabilities required for the FEL we provided by a permanent magnet-laced electromagnetic wiggler with a $10-\mathrm{cm}$ period and an overall length of $5.5 \mathrm{~m}$.

\footnotetext{
"Pefformed jointly under the euspices of the US DOE by LUNL under W-7405-ENG-48 and for the DOD under SDIO/SDC MIPR No. W43-GBL-0-5007.
}

\section{ENERGY SWEEP TOLERANCES}

Variation of beam energy will affect the FEL outpul power by two different mechanisms: through gain change as the beam energy deviates from resonance with the wiggler, and through energy sensitivity of the elignment of the beam with the axis of the wiggler. Sensitivity of beam power to off resonance operation has been calculated using the free electron laser simulation code. FRED [3,4]. For code input parameters in the expected operating range FRED predicts a $1 \%$ deviation from resonance energy reduces output power by $-6 \%$, $2 \%$ deviation by $-25 \%$.

Initial misalignmest of the bean with respect to the solenoidal guide field of the soceleratar will result in the beam centroid following helical path through the accelerator. Localized field errors will introduce jumps in the guidingcenter radius and position along the way. If the energy is cotstant the beam path will be fixed in space but energy variation will modulate the cyclotron wavelength and produce a complicaled sweeping in time of the beam centroid position and angle a any point including the entrance to the wiggler. This behovior, called beam "corkscrew" motion [S]-[B], imposes stringent requirements on mugnetic alignment and energy sweep.

In eartier operation two beam position monitors seperated by a field free region were used to measure the spatial and angular sweep at the exit of the eccelerator as the energy yaried. A bean unuspon code was used to calculate the corresponding motion at the entrance to the wiggler and these values were used as inpul into FRED to determine their effect on the microwave power. For the measured corkscrew motion we estimated that a $\pm 1 \%$ energy variation could decrease the ouput power by $30 \%$, much Irger effect than merely being off resonance. Considerable improvement in magnetic alignmen and reduction of corkscrew amplitude bave been made since the above measurements [7] but the goal of keeping the energy variation within $\pm 1 \%$ is still desirable. Maximizing the average FEl output power requires that the beam energy stay within these limits for a large fraction of the current pulse length. As a technology demonstration ow immediale milestone has been to maintain the energy sweep within $\pm 1 \%$ for at least $30 \mathrm{~ns}$.

\section{ENERGY SWEEP STUDIES}

\section{A. Beam energy measurement}

Our primary beam energy diagnostic has been a magnetic spoctrometer [1]. A bending magnet, located in the transport 
section between the accelerator and the wiggler, when energized, deflects the beam into a $45^{\circ}$ side am. Two peirs of beam position monitors, one on the input side and the other coaxial with the side arm are used to measure the variation of the deflection angle of the beam centroid in the bending plane around the $45^{\circ}$ central angle. The system can detect angular variations due to energy changes of $-0.1 \%$ and has a $500 \mathrm{MHz}$ bandwidth. The absolute energy calibration is derived from the mapping of the bending magnet's field.

\section{B.Sources of energy variation}

Energy variation may have either operational causes or be intrinsic to the design. Operational causes are those which affect shot reproducibility such as input voltage variation, timing jiner, veriation of ferrite resel condition, and insulator breakdown. Such uncontrolled variation can make the accelerator almost impossible to tune and virtually useless. Two important additions were made to the ETA-Il control system for the experiments described here, the first being a computer controlled feedback timing compensation system that correits for timing drifts due to power supply variations and the second an are and overvoltage protection system. which intermuts accelerator operation when a fault occurs. The latter along with the multicable feed system modification, described below, limit the energy available for driving an are and causing insulator damage. These changes greatly reduced uncontrolled variations and improved our ability to tune the machine. Remaining as problems are the intrinsic causes of energy variation within a stot -- time varying beam loading and cell impedence, and mismatches in the pulse-power feeds to the accelerator cells.

Measurements of beam energy variation at the output of ETA-II in its original configuration showed that the energy was within the $\pm 1 \%$ limits for at most 13 ns [1], less than hall of the period we could accept as a minimum. The source of most of this variation was traced to the cell pulse-power feeds. As originally configured, each ven-eell sel was fed from its magnetic pulse compressor (MAG-1D) by a single $4 \Omega$ water dielectric cable which connected to the input ends of a pair of busbars running parallel to the axis on opposite sides of the cell block. These provided a symerical power feed to the individual cells which tapped into the bars along the way. The far ends of the bars were terminated to reduce reflections. Although this design provided a neat mechanical solution to the problem of pulse-power disuribution, measurement and analysis soon showed that electrically it was not satisfactory. The busbars form a slow wave structure, consequently the phase of the vollage pulse with respect to the bean pulse $(\beta-1)$ varies with distance along the busbar. " "i.ce the beam loading modifies the applied volage pulse, phase variation results in each gap seeing a different resultant voltage variation with time and since the final beam energy is the sum of the contributions of all of the gaps, it is not surprising that a large energy swep was encountered.

\section{Modeling the power feed.}

A computer model of the pulse power feed was used to help understand its operation [9]. Experimental measurements of the injector current pulse shape, the MAG-1D voltage pulse shape and the variation of the cell lestage current with time were combined with a model of the busbar slow wave structure to calculate the time variation of each gaps voltage relative to the current pulse. The voltages were summed to give an estimate of the energy sweep of the output. The results of such a simulation are shown in Figure 1. We see that there is good agreement between the model's predictions and the experimental measurements of $\pm 1 \%$ energy sweep for a

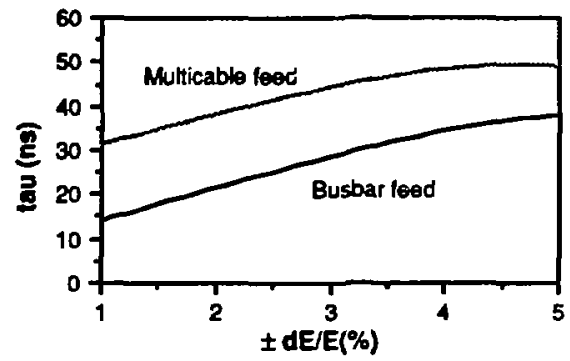

Figure 1. Time that besm energy stays within regulation range versus range (Simulation, 60 cells. E=6 Mev, $I=2 \mathrm{kA}$ )

maximum of 13ns with the old bushar feed.

Both experiment and modeling having shown that there was very little chance of meeting our milestone with the busbar power feed, the design of a new, multicable feed was undertaken. Such a feed system for the cells, while mechanically much more complex, allows trensit time isolation from the other cells while proper choice of cable lengths inșures constant phase betwoen curreti. pulse and gap voltages. Since it is best to feed the gaps symmetrically at two points, $180^{\circ}$ apart, our epprosch was to use two $40 \Omega$ solid dielectric cables to feed two cells in parallel. A short busbar on each side connects the high voltage electrodes of the cell pair and a cable connects to each busbar center. With this design there is -1 ns phase difference between the two cells relative to the current pulse and some sloshing around of energy between the cell pairs during the current rise and fall when the load is not matched. Modeling of this system. detailed in reference [9], showed that this new design should enable us to meet our energy sweep milestone for the full machine for certain conditions of current, MAG-1D voltage, and timing.(Figure 1).

\section{ETA-/I tests.}

On a tesi stand we developed a multicable feed system which would require minimum modification of the existing system. Each MAG-1D has two $4 \Omega$ output cables and can 
feed two ten-cell blocks. Rather than adapting the MAG-1D to the multicable feed, which would thave been a major lask, we installed a high voltage distribution box where transition was made from the two $4 \Omega$ cables to twenty $40 \Omega$ cables in parallel. The old busbar structure was replaced with the cell feed structure described above. After initial tests the new feed system was installed on the first two ten-cell blocks of ETA-II for evaluation before modifying the remainder of the aceelenuo. The remaining four ten-cell blocks were removed and reploced with a transport section, and the injector, whose design concentrates its voltage across the A-K gap, was left unchanged. This shortened version of ETA-II was capable of producing a 2.5-3 Mev beam.

The model was used to predict optimum values of MAGID voltage and relative timing between the injector and the accelerator for minimizing the energy sweep of a $-1.5 \mathrm{kA}$ beam from the accelerator. Experimental measurements agreed well with predictions of the model. If we define $\tau$ as the time the beam energy has a maximum peak $\omega$ peak variation of $2 \%$, then we were able to find conditions of voltage and timing delay near the model values for which $\tau$ exceeded $40 \mathrm{~ns}$. The best recorded shot is shown in Figure 2. While this shot is on the upper edge of the tau distribution the probability of

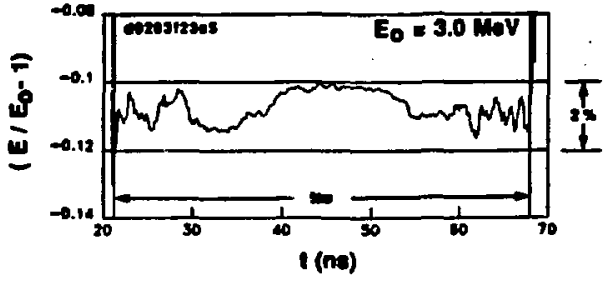

Figure 2. Beam energy variation vercus time (1 - $1.5 \mathrm{kA})$

exceeding the 30 ns milestone for these operating conditions was very high. Figure 3 is a histogram of the $\tau$ distribution for a set of 50 shots with the same input conditions. This data

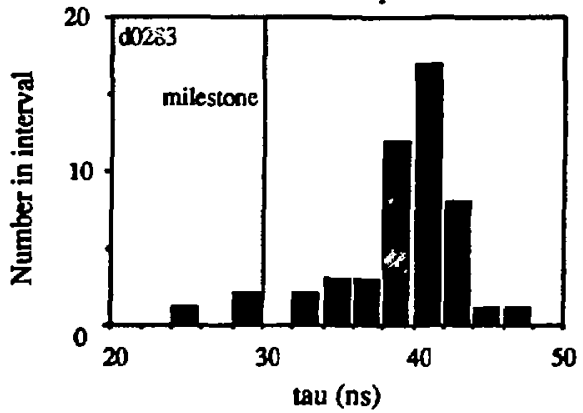

Figure 3. Histogram of un distribution for a set of 50 shots.

was recorded as 10 shot sets at various times during a days operation. Over this period of operation the drift of the central energy value was less than $1 \%$ (Figure 4)

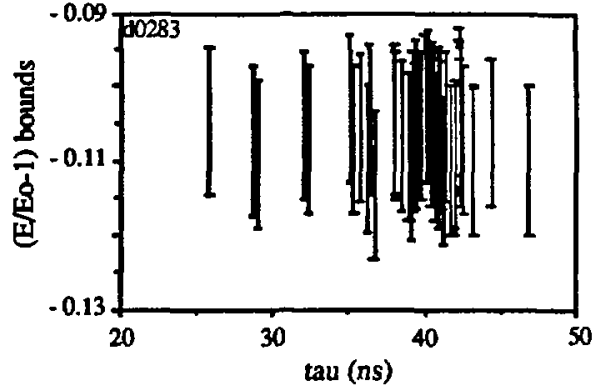

Figure 4. Energy bounds versus tau for date tel.

\section{SUMMARY}

In recent operation of a shortened version of ETA-II with only the injector and the first two cen-cell blocks we have been able to keep the energy sweep of the output beam to less than 2\% for periods greater than $\mathbf{4 0} \mathrm{ns}$. The key ingredient of this achievement was the retrofit of a new multicable pulse power foed to the 20 cells and the development of a model for predicting optimum operating parameters. This success was perhaps a necessary but not a sufficient condition to guarantee that we will be able to maintain the same degree of regulation at higher current and with the addition of the remaining four ten-cell blocks; however, the model predicts that a retrofit of the remaining ten-cell blocks with the new feeds will lead to the desired energy regulation at full energy and higher currents.

[1] W.E. Nexsen et al., The ETA-II Inducion Lirec as a HighAverage-Power FEL Driver," Nucl. Instr. and Mech., 1296 (1990) 54.61 .

[2] R.A. Jong et al., "TMP, A Free Electron Leset Amplifier for Plusma Henting in the Microwne Totanak Experiment," Nucl. Instr. and Mech.,A285 (1989) 379-386.

[3] T.J. Orzechowski et al., "High-Gin Free Electron Lesers using Induction Liseu Accelera:ors," IEEE J. Quantum Electron. QE-21 (1985) 831-844.

[4] E.T. Scharlemann et al., "Comparison of the Livermore Microwave FEL Results at ELF with 2D Numerical Simulation." Nucl. Instr. and Meth.,A250 (1986) 150-158.

[5] G.J. Caparso et a., Beam Dynumics in the Advanced Test Accelerator (ATA)," in Proc. Sth High Power Particle Beams Conf., San Francisco, CA, September, 1983,pp. 427-432.

[6] Y.J. Chen, "Corkscrew Modes in Linear Accelerators," Nucl. Instr. and Meth., A292 (1990) 455-464.

[7] S.L. Allen et el., "Messurements of Reduced Corkscrew Motion on the ETA-II Lineur Induction Accelerator." these proceedings.

[8] W.C. Tumer, "Control of Energy Sweep and Transverre Beam Motion in Induction Linacs," these proceedings.

[9] S.E. Sumpayan et al., "Energy Sweep Compensation of Induction Accelerstors." in Proc. 1990 Linear Accel. Conf., Albuquerque, NM, September, 1990. 